\title{
Efeitos dos Contraceptivos Hormonais Orais de Baixa Dosagem Estrogênica nas Taxas de Folato Intra-eritrocitário
}

\author{
Effects of Low-Dose Oral Hormonal Contraceptive on Intraerythrocytic Folate Levels \\ Túlio Bráulio Cantalice de Paula ${ }^{1}$, Antonio Fernandes Moron ${ }^{1}$, Cristina Guazzelli², \\ Kimyio Nonoyama ${ }^{3}$, Cristiani Martinez Salzone ${ }^{3}$
}

\begin{abstract}
RESUMO
Objetivo: identificar os efeitos do uso dos contraceptivos hormonais orais (ACHO) de baixa dosagem estrogênica ( $<30 \mu \mathrm{g}$ de etinilestradiol) nas taxas intra-eritrocitárias de folatos. Métodos: este foi estudo prospectivo transversal que incluiu 95 pacientes atendidas no Ambulatório de Planejamento Familiar da Escola Paulista de Medicina - UNIFESP. O grupo controle (grupo Condom) foi constituido por pacientes usuárias de condom como método contraceptivo exclusivo nos últimos doze meses e os grupos de estudo eram compostos por usuárias de ACHO de baixa dosagem agrupadas de acordo com o tempo de uso do método, quer seja, grupo ACHO 3 (três a seis meses de uso), grupo ACHO 6 (seis a doze meses de uso) e grupo ACHO 12 (mais de doze meses de uso). A dosagem de folatos intra-eritrocitários foi feita pelo método de captura iônica. A avaliação estatística dos resultados foi feita pela análise de variância e teste $\chi^{2}$.

Resultados: no grupo Condom observou-se que 44\% das pacientes apresentaram niveis de folato intra-eritrocitário reduzidos ( $<186,0 \mathrm{ng} / \mathrm{mL})$, ao passo que nos grupos de usuárias de ACHO este indice foi de 32\% (grupo ACHO 3), 16\% (grupo ACHO 6) e 31\% (grupo ACHO 12). Não se encontrou nos grupos de usuárias de ACHO de baixa dosagem estrogênica redução significativa na média das taxas de folato intra-eritrocitário em relação ao grupo controle, nem houve diferença dos resultados em função do tempo de uso do método.

Conclusões: verificaram-se niveis reduzidos de folato intra-eritrocitário numa parcela significativa (44\%) de pacientes não usuárias de ACHO, com valores próximos ao limite inferior do que é considerado normal pela maioria dos autores, traduzindo deficiência basal de folatos na população estudada. Não se observou influência do uso de ACHO de baixa dosagem estrogênica sobre as taxas de folato intra-eritrocitário.
\end{abstract}

PALAVRAS-CHAVE: Contracepção. Condom. Contraceptivos orais.

${ }^{1}$ Disciplina de Medicina Fetal/Escola Paulista de Medicina/ Universidade Federal de São Paulo

${ }^{2}$ Disciplina de Obstetrícia Fundamental/Escola Paulista de Medicina/Universidade Federal de São Paulo

${ }^{3}$ Secretaria de Saúde do Estado de São Paulo: Instituto Adolfo Lutz

Túlio Bráulio Cantalice de Paula

Rua João Fragoso de Medeiros, n 2428, apto. 1401 - Bairro Candeias

54430-250 - Jaboatão dos Guararapes - PE

Fone: (81) 3091-3074, (81) 3474-0331, (81) 9972-3331/

Fone/Fax: (11) 5572-4449

e-mail: Raqueleta2002@yahoo.com.br mariarachelviegas@terra.com.br
Introdução

Os folatos são coenzimas necessárias para a multiplicação celular, participando da sintese das purinas e pirimidinas na replicação do DNA, do ciclo homocisteína-metionina e do metabolismo dos aminoácidos glicina, serina e histidina, além do metabolismo das aminas ${ }^{1}$.

Entre as deficiências vitamínicas, a dos folatos é a mais comum entre as mulheres norte-americanas, causando alterações hematológicas em 15 a 
$30 \%$ das gestantes. Em estudo realizado na cidade de São Paulo observou-se que $12,2 \%$ das gestantes eram deficientes em folatos e $6,5 \%$ apresentavam anemia associada a esta deficiência. Ressalta-se, ainda, que a causa mais comum da redução dos níveis de folato é a baixa ingestão desta vitamina ${ }^{2}$.

Os seres humanos não são capazes de sintetizar folatos, o que os torna dependentes das fontes alimentares ${ }^{3,4}$. O aporte adequado de folatos pode ser feito pela ingestão de vegetais frescos, frutas cítricas, figado, feijão, nozes, pão integral e leguminosas, pela suplementação vitamínica ou pela ingestão de alimentos enriquecidos com ácido fólico, tais como farináceos ${ }^{5}$.

As concentrações teciduais de folato refletem mais fielmente as suas reservas do que os níveis séricos. A dosagem de folato sérico representa a ingesta deste elemento na última semana (ingestão recente), enquanto que o folato intraeritrocitário representa a real concentração tecidual (ingestão crônica) ${ }^{6}$. O único tecido utilizado na prática para a dosagem de folatos é o sangue, mais precisamente os eritrócitos, que são menos sujeitos a flutuações de suas taxas ${ }^{7}$. Os folatos são incorporados aos eritrócitos durante a eritropoese, refletindo com maior precisão o estado corpóreo das reservas de folato ${ }^{8}$.

A influência dos contraceptivos hormonais orais (ACHO) no metabolismo dos folatos é assunto bastante controverso e tema de estudos desde a década de 60 . Shojania et al. ${ }^{9}$ foram os primeiros a relatar a deficiência de folatos séricos e intra-eritrocitários em usuárias de contraceptivos hormonais orais. Mostraram, ainda, que estas alterações desaparecem logo após a suspensão do método.

Os ACHO podem reduzir os niveis de folato sérico e intra-eritrocitário por meio de interferência na absorção do folato contido nos alimentos, aumento da sua excreção urinária e indução de enzimas hepáticas (aumento da metabolização), além do aumento das proteínas séricas ligadoras de folatos. A inibição da conjugase intestinal reduz a absorção dos folatos poliglutâmicos da dieta em aproximadamente $50 \%$, porém não altera a absorção dos folatos monoglutâmicos ${ }^{10}$.

Sabe-se que o uso de ACHO durante os três meses que antecedem a gestação aumenta o risco de defeitos de fechamento do tubo neural (DFTN), fendas faciais e anomalias cromossômicas $^{11}$. Verificou-se que o uso periconcepcional de multivitamínicos com ácido fólico reduz o risco de lábio leporino com ou sem fenda palatina ${ }^{12}$, defeitos cardíacos e dos membros ${ }^{13}$ e estenose hipertrófica de piloro ${ }^{14}$.

Não é conhecida a relação entre o tipo e a dosagem hormonal utilizados nos contraceptivos e seus efeitos nas reservas intra-eritrocitárias de folatos, uma vez que os estudos mais amplos disponiveis envolveram doses elevadas de etinilestradiol $^{15}$.

O nível de folato intra-eritrocitário considerado normal em adultos varia de 160 a $640 \mathrm{ng} / \mathrm{mL}$, considerando-se deficiente quando inferior a 140 $\mathrm{ng} / \mathrm{ml}$. Durante a gestação, consideram-se normais os valores de 200 a $800 \mathrm{ng} / \mathrm{mL}$ para as mães e de 400 a $1350 \mathrm{ng} / \mathrm{mL}$ para o feto ${ }^{16}$.

Alguns estudos ${ }^{17}$ mostram que o risco para DFTN em gestantes com folato intra-eritrocitário entre 200 a $299 \mathrm{ng} / \mathrm{mL}$ na época do fechamento do tubo neural é três vezes maior e quando estes níveis estão abaixo de $150 \mathrm{ng} / \mathrm{mL}$ o risco é oito vezes maior comparado a gestantes com folato intra-eritrocitário acima de $400 \mathrm{ng} / \mathrm{mL}$.

Para mulheres que interromperam o uso de $\mathrm{ACHO}$ nos últimos três meses antes de engravidar ou que continuaram a sua utilização durante o início da gestação, o risco para DFTN é de 1,18 quando comparado com mulheres que nunca tomaram contraceptivos orais ${ }^{18}$.

Para preservar a saúde do binômio mãe-feto, no contexto da Saúde Pública, a investigação sobre os efeitos dos ACHO contendo baixas doses de etinilestradiol nas taxas de folato intraeritrocitário deve ser valorizada, pois persistem questionamentos importantes para uma adequada orientação sobre a prevenção de anomalias fetais associadas às deficiências nutricionais e exposição a medicamentos. O presente estudo teve como objetivo estudar os efeitos dos contraceptivos hormonais orais de baixa dosagem estrogênica $(<30 \mu \mathrm{g})$, ou seja, das modernas pilulas anticoncepcionais, sobre as reservas intra-eritrocitárias de folato.

\section{Pacientes e Métodos}

Este foi um estudo prospectivo transversal aprovado pela Comissão de Ética em Pesquisa da Escola Paulista de Medicina - UNIFESP, que incluiu 95 pacientes divididas em quatro grupos. $\mathrm{O}$ grupo controle (grupo Condom foi constituído por pacientes que optaram pelo uso do condom como método contraceptivo exclusivo nos últimos doze meses, sem que possuíssem qualquer problema clínico ou contra-indicação para o uso de contraceptivos hormonais. Os demais grupos eram compostos por pacientes que usavam $\mathrm{ACHO}$ de baixa dosagem $(<30 \mu \mathrm{g}$ de etinilestradiol) por um período de três a seis meses (grupo ACHO 3), seis a doze meses (grupo ACHO 6) e mais de doze meses (grupo ACHO 12). Todas as pacientes eram acompa- 
nhadas no Setor de Planejamento Familiar da Disciplina de Obstetrícia/EPM-UNIFESP. A participação no presente estudo foi voluntária, realizandose a coleta de amostra de sangue venoso periférico $(5 \mathrm{~mL})$ no período da manhã, após minuciosa explicação sobre o estudo e conseqüente assinatura do consentimento informado.

Foram incluídas no estudo apenas u suárias de ACHO de baixa dosagem estrogênica $(<30 \mu \mathrm{g}$ de etinilestradiol) ou condom como método contraceptivo exclusivo, com idade de 18 a 28 anos. O tipo de progesterona (desogestrel, gestodeno ou levonorgestrel) contido na pílula não foi considerado como critério discriminativo. As pacientes não foram classificadas de acordo com o índice de massa corporal, raça, paridade ou antecedente de filho com malformação congênita.

Os critérios de exclusão foram: pacientes com hipermenorragia ou hipermenorréia, anemia ( $\mathrm{Hb}<10,5 \mathrm{~g} / \mathrm{dL})$, usuárias de DIU, portadoras de doenças hematológicas, endocrinopatias, doenças auto-imunes, coagulopatias, curto intervalo após o último parto ( $<2$ anos), usuárias de outras medicações, tabagistas e etilistas.

A dosagem de folatos intra-eritrocitários foi feita pelo método de captura iônica na Seção de Hematologia, Divisão de Patologia do Instituto Adolfo Lutz da Secretaria de Saúde do Estado de São Paulo. O ensaio IMX Folatos (Abbott) utiliza reagente de afinidade solúvel composto por uma proteína de ligação aos folatos (FBP) ligada a anticorpos monoclonais, que por sua vez têm uma ligação covalente com a carboximetilamilose. Os complexos de analitos com carga negativa são formados durante o ensaio IMX Folatos pela ligação entre os folatos e o reagente de afinidade solúvel. Os complexos de analitos de carga negativa resultantes são então capturados por meio da interação eletrostática com a matriz de carga positiva. Os folatos são quantificados medindo a quantidade de locais de união da FBP ligados a matriz que não foram ocupados pelo analito utilizando um conjugado de ácido pteróico (um análogo dos folatos) e fosfatase alcalina como molécula geradora do sinal. A matriz é lavada de forma a remover o conjugado não ligado. O substrato 4-metilumbeliferilfosfato é adicionado à matriz e o produto fluorescente é medido pelo conjunto óptico MEIA.

A tecnologia MEIA (enzimaimunoensaio por micropartículas) tem como princípio o uso de micropartículas sensibilizadas com anticorpos ou antígenos (dependendo da finalidade do produto), sendo sua técnica utilizada para testes qualitativos e quantitativos. O fato de esta nova tecnologia utilizar micropartículas como fase sólida aumenta a sensibilidade e a especificidade do método e diminui o seu tempo de incubação. Isto se deve ao número de sítios de ligações de um teste por micropartículas ser de 4000 a 10000 vezes maior do que o EIA (enzimaimunoensaio) tradicional.

A análise estatística baseou-se na análise de variantes múltiplas e teste $t$ de Student, com um poder de amostragem superior a 0,80 e nível de significância em $\mathrm{p}<0,05$ (nível de rejeição de hipótese). Os grupos ACHO 3, ACHO 6 e ACHO 12 foram comparados entre si e com o grupo controle (grupo Condom).

Os resultados obtidos também foram comparados com um estudo ${ }^{16}$ envolvendo 118 indivíduos saudáveis, que encontrou concentração média de folatos intra-eritrocitários de $297,5 \mathrm{ng} / \mathrm{mL}$, com intervalo de 111,6 a $761,5 \mathrm{ng} / \mathrm{mL}$. Utilizando análise não-paramétrica, verificamos que 95\% da população apresentava resultados de 148,8 a 645,4 $\mathrm{ng} / \mathrm{mL}$, definindo como limite inferior da faixa de normalidade o valor de $186 \mathrm{ng} / \mathrm{mL}$.

\section{Resultados}

A dosagem das taxas de folato intraeritrocitário das 95 pacientes estudadas revelou que $69,5 \%$ destas apresentavam niveis de folato intra-eritrocitário acima do limite inferior da normalidade (>186 $\mathrm{ng} / \mathrm{mL}$ ), ao passo que em $30,5 \%$ dos casos encontraram-se níveis de folato inferiores ao normal.

A população utilizada como controle (grupo Condom), composta por pacientes que optaram pelo uso do condom como método contraceptivo exclusivo nos últimos doze meses, apresentou $44 \%$ de pacientes com folato intra-eritrocitário reduzido $(<186,0 \mathrm{ng} / \mathrm{mL})$, ao passo que nos grupos de estudo, compostos por pacientes que usavam $\mathrm{ACHO}$ de baixa dosagem estrogênica $(<30 \mu \mathrm{g}$ de etinilestradiol) por um período de três a seis meses (grupo ACHO 3), seis a doze meses (grupo ACHO 6) e mais de doze meses (grupo ACHO 12), este indice foi de 32, 16 e 31\%, respectivamente. Contrariando estudos anteriores ${ }^{6,7}$ sobre a influência dos ACHO sobre as taxas de folato intraeritrocitário, não se encontrou nas usuárias de ACHO de baixa dosagem estrogênica uma redução na média das taxas de folato intraeritrocitário (Tabelas 1, 2).

A Tabela 3 mostra a distribuição de pacientes com valores de folato intra-eritrocitário inferiores ao normal. Observa-se que o grupo Condom apresenta $38 \%$ das 29 pacientes com taxas reduzidas de folato intra-eritrocitário encontradas durante o estudo. Do total de pacientes estudadas, $83 \%$ apresentaram valores abaixo de $299 \mathrm{ng} / \mathrm{mL}$. 
Tabela 1 - Distribuição de pacientes segundo niveis de folato intra-eritrocitário nos grupos estudados.

\begin{tabular}{lccc} 
& \multicolumn{3}{c}{ Nível de folato } \\
& Normal n (\%) & Reduzido $\mathbf{n}(\%)$ & Total $\mathbf{~}$ \\
\hline Grupo Condom & $14(56)$ & $11(44)$ & 25 \\
Grupo ACHO 3 & $13(68)$ & $6(32)$ & 19 \\
Grupo ACHO 6 & $21(84)$ & $4(16)$ & 25 \\
Grupo ACHO 12 & $18(69)$ & $8(31)$ & 26 \\
Total & $66(69,5)$ & $29(30,5)$ & 95 \\
\hline
\end{tabular}

Nota: Grupos ACHO 3, 6 e 12: pacientes em uso de contraceptivo oral por 3, 6 ou 12 meses.

Tabela 2 - Freqüência, média, desvio padrão (DP) e valores mínimo e máximo para as taxas de folato intra-eritrocitário nos grupos estudados.

\begin{tabular}{lcccc}
\hline & $\begin{array}{c}\text { Freqüência } \\
\mathbf{n}\end{array}$ & $\begin{array}{c}\text { Média } \pm \mathrm{DP} \\
(\mathbf{n g} / \mathrm{mL})\end{array}$ & $\begin{array}{r}\text { Mínimo } \\
(\mathbf{n g} / \mathrm{mL})\end{array}$ & $\begin{array}{c}\text { Máximo } \\
(\mathbf{n g} / \mathrm{mL})\end{array}$ \\
\hline Grupo Condom & 25 & $243,9 \pm 141,8$ & 113,0 & 800,9 \\
Grupo ACHO 3 & 19 & $208,2 \pm 56,3$ & 133,6 & 307,3 \\
Grupo ACHO 6 & 25 & $274,6 \pm 130,3$ & 143,4 & 780,0 \\
Grupo ACHO 12 & 26 & $233,6 \pm 99,3$ & 97,7 & 554,6 \\
Total & 95 & $242,0 \pm 115,0$ & 121,9 & 610,7
\end{tabular}

Nota: Grupos ACHO 3, 6 e 12: pacientes em uso de contraceptivo oral por 3, 6 ou 12 meses.

Tabela 3 - Distribuição de pacientes com valores de folato intra-eritrocitário inferiores ao normal nos grupos estudados. Valores medidos em cada grupo e desvio padrão.

\begin{tabular}{lcc}
\hline & $\begin{array}{c}\text { Freqüência } \\
\mathbf{n}(\%)\end{array}$ & $\begin{array}{c}\text { Média } \\
(\mathbf{n g} / \mathbf{m L})\end{array}$ \\
\hline Grupo Condom & $11(38)$ & $154,0(59,7)$ \\
Grupo ACHO 3 & $6(20)$ & $139,7(72,0)$ \\
Grupo ACHO 6 & $4(14)$ & $149,6(48,8)$ \\
Grupo ACHO 12 & $8(28)$ & $140,8(66,3)$ \\
Total & $29(100)$ & $146,0(61,7)$ \\
\hline
\end{tabular}

Nota: Grupos ACHO 3, 6 e 12: pacientes em uso de contraceptivo oral por 3, 6 ou 12 meses.

\section{Discussão}

Os primeiros relatos da deficiência de folatos séricos e intra-eritrocitários em usuárias de ACHO surgiram na década de sessenta. Mostraram, também, que estas alterações desaparecem logo após a suspensão do contraceptivo e são de ocorrência rara. De acordo com os primeiros estudos $^{9,18}$, os contraceptivos orais interferem na absorção e metabolismo dos folatos.

Em um estudo envolvendo 118 indivíduos saudáveis ${ }^{16}$, encontrou-se concentração média de folatos intra-eritrocitários de $297,5 \mathrm{ng} / \mathrm{mL}$, com intervalo de 111,6 a $761,5 \mathrm{ng} / \mathrm{mL}$. Utilizando análise não-paramétrica, estes autores verificaram que $95 \%$ da população apresentava resultados de 148,8 a $645,4 \mathrm{ng} / \mathrm{mL}$, definindo como limite inferior da faixa de normalidade o valor de $186 \mathrm{ng} / \mathrm{mL}$.
Estudo recente envolvendo o uso de $\mathrm{ACHO}$ contendo baixas doses de etinilestradiol $(<30 \mu \mathrm{g})$ mostrou que pode haver minimização da redução de folatos com esta dosagem do componente estrogênico ${ }^{3}$. Nossos resultados não mostraram redução nas taxas de folato intra-eritrocitário de usuárias de ACHO de baixa dosagem estrogênica ao serem compararadas com aquelas observadas no grupo Condom, mesmo após 12 meses de uso das pílulas. Paralelamente, observaram-se niveis reduzidos $(<186,0 \mathrm{ng} / \mathrm{mL})$ de folato intraeritrocitário em $44 \%$ das pacientes do grupo Condom, sendo a média dos valores deste grupo de $243,9 \mathrm{ng} / \mathrm{mL}$. Isto nos alerta para a presença de deficiência basal de folatos na população estudada, seja por um componente nutricional isolado ou associado ao fator genético, podendo ter sido suficiente para mascarar o efeito intrínseco do uso dos ACHO de baixa dosagem sobre o metabolismo e reserva de folatos.

Não se encontrou nos grupos de usuárias de ACHO de baixa dosagem estrogênica deficiência nas taxas de folato intra-eritrocitário maior que no grupo controle (grupo Condom), o que se traduz em ausência de inibição significativa da atividade da enzima conjugase intestinal com o uso de doses reduzidas do componente estrogênico nas pílulas, contrariando estudos ${ }^{10,19}$ que avaliaram usuárias de pilulas com maior dosagem estrogênica (>30 $\mu \mathrm{g})$.

Nosso estudo revelou dados importantes sobre os reduzidos niveis de folato na população investigada, mostrando que $83 \%$ das pacientes apresentam taxas de folato intra-eritrocitário inferiores a $299,0 \mathrm{ng} / \mathrm{mL}$, colocando esta população em um grupo de risco três vezes maior para DFTN quando se compara com pacientes que apresentam niveis de folato intra-eritrocitário superiores a $400 \mathrm{ng} / \mathrm{ml}^{17}$.

Comparando-se os grupos de usuárias de $\mathrm{ACHO}$ de baixa dosagem estrogênica (grupos ACHO 3 , ACHO 6 e ACHO 12) em função do tempo de uso do método com o grupo controle (grupo Condom), não se observou diferença estatisticamente significativa nas taxas de folato intra-eritrocitário, podendo esta dose reduzida do componente estrogênico ser incapaz de interferir na absorção do folato da dieta, na sua excreção urinária e metabolização hepática ou na indução do aumento da sintese das proteínas ligadoras de folatos.

Alguns autore ${ }^{10}$ tentam justificar a queda dos níveis de folato intra-eritrocitário em usuárias de ACHO como sendo devida a uma restrição global da ingestão de alimentos visando evitar o ganho de peso inerente ao uso de hormônios esteróides. Porém, as modernas pílulas com dosagens estrogênicas minimas e derivados progestagêni- 
cos de última geração (gestodeno e desogestrel) não apresentam os efeitos de retenção hídrica e conseqüente ganho ponderal, portanto deixando de constituir fator que impulsione a mudança do padrão alimentar das usuárias, podendo-se manter a ingestão habitual de folatos na dieta.

\section{ABSTRACT}

Purpose: to study the effects of low-dose oral hormonal contraceptives $(\mathrm{OHC})(<30 \mu \mathrm{g}$ of ethynylestradiol) on the intraerythrocytic folate levels.

Methods: this was a prospective transversal study with 95 patients treated in the Family Planning Clinic of UNIFESP (Federal University of São Paulo). The control group (Condom group) consisted of patients using condom as their exclusive contraceptive method during the last 12 months, and the study groups consisted of patients using low-dose oral hormonal contraceptives, in the following way: $\mathrm{OHC}$ 3 group (three to six months of use), OHC 6 group (six to twelve months of use) and OHC 12 group (more than twelve months of use). Intraerythrocytic folate was determined by the ionic capture method. Analysis of variance and $\chi^{2}$ test were used for statistical analysis.

Results: the Condom group showed a rate of $44 \%$ of patients with folate lower than $186.0 \mathrm{ng} / \mathrm{mL}$ and the users of lowdose oral contraceptives showed a rate of $32 \%(\mathrm{OHC} 3$ group), $16 \%$ (OHC 6 group) and 31\% (OHC 12 group). We did not find in the group using low-dose oral contraceptives a significant reduction in the average level of intraerythrocytic folate compared to the control group and there was no statistically significant difference $(p=0.28)$ regarding time of use.

Conclusion: we observed reduced levels of intraerythrocytic folate in a significant number (44\%) of patients not using low-dose oral hormonal contraceptives. Their rates were similar to the lower limit considered to be normal by most authors, which points to a basal folate deficiency in the studied group. We did not observe any alteration in the level of intraerythrocytic folate in patients using low-dose oral hormonal contraceptives.

KEYWORDS: Contraceptives. Condom. Intraerythrocytic folate.

\section{Referências}

1. Hibbard BM. Folates and fetal development. $\mathrm{Br} \mathrm{J}$ Obstet Gynaecol 1993; 100:307-9.

2. Thame G, Shinohara EMG, Salsone CM, Santos JC, Uehara DY, Moron AF. Determinação de valores de ácido fólico sérico em gestantes com até 22 semanas de gestação. Newslab 1996; 4:57-61.

3. Steegers-Theunissen RP, Smithells RW, Eskes TK. Update of new risk factors and prevention of neural tube defects. Obstet Gynecol Surv 1993; 48:287-93.
4. Rose NC, Mennuti MT. Periconceptional folic acid supplementation as a social intervention. Semin Perinatol 1995; 19:243-54.

5. Organización Mundial de la Salud. Anemias nutricionales: informe de um grupo de expertos de la OMS. Ginebra; 1972. (Série de Informes Técnicos, 503).

6. Smithells RW, Nevin NC, Seller MJ, et al. Further experience of vitamin supplementation for prevention of neural tube defect recurrences. Lancet $1983 ; 1: 1207-31$

7. Bunduki V, Dommergues M, Zittoun J, Marquet J, Muller F, Dumez Y. Maternal-fetal folate status and neural tube defects: a case control study. Biol Neonate 1995; 65:154-9.

8. van der Put NM, Thomas CM, Eskes TK, et al. Altered folate and vitamin $B_{12}$ metabolism in families with spina bifida offspring. QJM 1997; 90:505-10.

9. Shojania AM, Hornady G, Barnes PH. Oral contraceptives and serum folate level. Lancet 1968; $1: 1376-7$

10.Lindenbaum J, Allen RH. Clinical spectrum and diagnosis of folate deficiency. In: Bailey LB, editor. Folate in Health and Disease. $1^{\text {st }}$ ed. New York: Marcel Dekker; 1995. p.43-73.

11. Carr DH. Chromosomes after oral contraceptives. Lancet $1967 ; 2: 830-1$.

12.Tolarova M. Periconceptional supplementation with vitamins and folic acid to prevent recurrence of cleftlip. Lancet 1983; 2:217.

13. Shaw GM, O'Malley CD, Wasserman CR, Tolarova MM, Lammer EJ. Maternal periconceptional use of multivitamins and reduced risk for conotruncal heart defects and limb deficiencies among offspring. Am J Med Genet 1995; 59:536-45.

14.Czeizel AE. Periconceptional folic acid containing multivitamin supplementation. Eur J Obstet Gynecol Reprod Biol 1998; 78:151-61.

15.Prevention of neural tube defects: results of the Medical Research Council Vitamin Study Research Group. Lancet 1991; 338:131-7.

16.Economides DL, Ferguson J, Mackenzie IZ, Darley $\mathrm{J}$, Ware II, Holmes-Siedle M. Folate and vitamin $\mathrm{B}_{12}$ concentrations in maternal and fetal blood, and amniotic fluid in second trimester pregnancies complicated by neural tube defects. Br J Obstet Gynaecol 1992; 99:23-5.

17.Daly LE, Kirke PN, Molloy A, Weir DG, Scott JM. Folate levels and neural tube defects: implications for prevention. JAMA 1995; 274:1698-702.

18.Lambie DG, Johnson RH. Drugs and folate metabolism. Drugs 1985; 30:145-55.

19.Richards ID. Congenital malformations and environmental influences in pregnancy. Br J Prev Soc Med 1969; 23:218-25.

Recebido em: 7/7/2003 Aceito com modificações em: 15/8/2003 